\title{
Two-dimensional nano-sheets produced by liquid exfoliation of layered materials
}

Jonathan N Coleman $^{1 *}$, Mustafa Lotya ${ }^{1}$, Arlene O’Neill ${ }^{1}$, Shane D Bergin ${ }^{1}$, Paul J King ${ }^{1}$, Umar Khan ${ }^{1}$, Karen Young ${ }^{1}$, Alexandre Gaucher ${ }^{1}$, Sukanta De ${ }^{1}$, Ronan J Smith, ${ }^{1}$ Igor V Shvets $^{1}$, Sunil K Arora ${ }^{1}$, George Stanton ${ }^{1}$, Hye-Young Kim ${ }^{2,3}$, Kangho Lee ${ }^{2,3}$, Gyu Tae $\mathrm{Kim}^{3}$, Georg S Duesberg ${ }^{2}$, Toby Hallam ${ }^{2}$, John $\mathrm{J}_{\text {Boland }}{ }^{2}$, Jing Jing Wang, ${ }^{1}$ John F Donegan, ${ }^{1}$ Jaime C Grunlan ${ }^{4}$, Gregory Moriarty ${ }^{4}$, Aleksey Shmeliov ${ }^{5}$, Rebecca J Nicholls ${ }^{5}$, James M Perkins ${ }^{6}$, Eleanor M. Grieveson ${ }^{5}$, Koenraad Theuwissen ${ }^{5}$, David W. $\mathrm{McComb}^{6}$, Peter D. Nellist ${ }^{5}$ and Valeria Nicolosi ${ }^{5^{*}}$

${ }^{1}$ School of Physics and CRANN, Trinity College Dublin, D2, Ireland

${ }^{2}$ School of Chemistry and CRANN, Trinity College Dublin, D2, Ireland

${ }^{3}$ School of Electrical Engineering, Korea University, Seoul, South Korea

${ }^{4}$ Department of Mechanical Engineering, Texas A and M University, College Station, Texas 77843, USA

${ }^{5}$ Department of Materials, University of Oxford, Parks Road, Oxford OX1 3PH, UK

${ }^{6}$ Department of Materials, Imperial College London, London, SW7 2AZ, UK

*colemaj@tcd.ie,valeria.nicolosi@materials.ox.ac.uk

\section{One sentence summary:}

We describe a general liquid-phase method to exfoliate layered compounds to give monoand few-layer flakes in large quantities. 


\begin{abstract}
If they could be easily exfoliated, layered materials would become a diverse source of 2-dimensional crystals whose properties would be useful in applications from electronics to energy storage. We show that layered compounds such as $\mathrm{MoS}_{2}, \mathrm{WS}_{2}$, $\mathrm{MoSe}_{2}, \mathrm{MoTe}_{2}, \mathrm{TaSe}_{2}, \mathrm{NbSe}_{2}, \mathrm{NiTe}_{2}, \mathrm{BN}$ and $\mathrm{Bi}_{2} \mathrm{Te}_{3}$, can be efficiently dispersed in common solvents and can be deposited as individual flakes or formed into films. Electron microscopy confirms the material to be exfoliated into individual layers. By blending with suspensions of other nano-materials or polymer solutions, we can prepare hybrid dispersions or composites which can be cast into films. We show that $\mathrm{WS}_{2}$ and $\mathrm{MoS}_{2}$ effectively reinforce polymers, while $\mathrm{WS}_{2} /$ carbon nanotube hybrid films have high conductivity leading to promising thermoelectric properties.
\end{abstract}


Layered materials represent a diverse and largely untapped source of 2-dimensional (2D) systems with exotic electronic properties and high specific surface areas that are important for sensing, catalysis and energy storage applications. While graphene is the most well-known layered material, transition metal dichalcogenides (TMDs), transition metal oxides (TMOs) and other 2D compounds such as $\mathrm{BN}, \mathrm{Bi}_{2} \mathrm{Te}_{3}$ and $\mathrm{Bi}_{2} \mathrm{Se}_{3}$ are also important. The latter materials are of particular interest as topological insulators and thermoelectric materials(1). However, development of these materials has been hampered by the lack of a simple method to exfoliate them to give mono- or few-layer flakes in large quantities.

TMDs consist of hexagonal layers of metal atoms, M, sandwiched between two layers of chalcogen atoms, $\mathrm{X}$, with stoichiometry $\mathrm{MX}_{2}$. While the bonding within these tri-layer sheets is covalent, adjacent sheets stack via van der Waals interactions to form a $3 \mathrm{D}$ crystal. TMDs occur in more than 40 different types $(2,3)$ depending on the combination of chalcogen ( $\mathrm{S}, \mathrm{Se}$ or $\mathrm{Te}$ ) and transition metal(3). Depending on the co-ordination and oxidation state of the metal atoms, TMDs can be metallic, semi-metallic or semiconducting $(2,3)$, e.g. $\mathrm{WS}_{2}$ is a semiconductor while $\mathrm{NbSe}_{2}$ is a metal(3). In addition, superconductivity(4) and charge density wave effects(5) have been observed in some TMDs. This versatility makes them potentially useful in many areas of electronics.

However, like graphene(6), layered materials must be exfoliated to fulfil their full potential. For example, films of exfoliated $\mathrm{Bi}_{2} \mathrm{Te}_{3}$ should display enhanced thermoelectric efficiency by suppression of thermal conductivity(7). Exfoliation of 2D topological insulators such as $\mathrm{Bi}_{2} \mathrm{Te}_{3}$ and $\mathrm{Bi}_{2} \mathrm{Se}_{3}$ would reduce residual bulk conductance, 
highlighting surface effects. In addition, we can expect changes in electronic properties as the number of layers is reduced e.g. the indirect bandgap of bulk $\mathrm{MoS}_{2}$ becomes direct in few-layer flakes(8). Although exfoliation can be achieved mechanically on a small $\operatorname{scale}(9,10)$, liquid phase exfoliation methods are required for many applications $(11)$. Critically, a simple liquid exfoliation method would allow the formation of novel hybrid and composite materials. While TMDs can be chemically exfoliated in liquids(12-14), this method is time consuming, extremely sensitive to the environment and incompatible with most solvents.

We demonstrate exfoliation of bulk TMD crystals in common solvents to give mono- and few layer nano-sheets. This method is insensitive to air and water and can potentially be scaled up to give large quantities of exfoliated material. In addition, we show that this procedure allows the formation of hybrid films with enhanced properties.

We initially sonicated commercial $\mathrm{MoS}_{2}, \mathrm{WS}_{2}$ and $\mathrm{BN}(15,16)$ powders in a number of solvents with varying surface tensions. The resultant dispersions were centrifuged and the supernatant decanted (Section S3). Optical absorption spectroscopy showed that the amount of material retained (characterised by $A / l=\alpha C$, where $\mathrm{A} / 1$ is the absorbance per length, $\alpha$ is the extinction coefficient and $\mathrm{C}$ is the concentration) was maximised for solvents with surface tension close to $40 \mathrm{~mJ} / \mathrm{m}^{2}(17,18)$ (Fig. 1A-C). Detailed analysis, within the framework of Hansen solubility parameter theory(19), shows successful solvents to be those with dispersive, polar and H-bonding components of the cohesive energy density within certain well-defined ranges (Section S4, Figs. S2-S3). This can be interpreted to mean that successful solvents are those which minimise the energy of 
exfoliation. Importantly, this information will facilitate the search for new solvents and the development of solvent blends. Some of the more promising solvents were N-methylpyrrolidone (NMP) and isopropanol (IPA)(see Table S1 for full solvent list). Optimization of the dispersion procedure (Section S5), gave concentrations as high as 0.3 $\mathrm{mg} / \mathrm{ml}$ for $\mathrm{MoS}_{2}, 0.15 \mathrm{mg} / \mathrm{ml}$ for $\mathrm{WS}_{2}$ (both in NMP) and $0.06 \mathrm{mg} / \mathrm{ml}$ for BN (IPA). Photographs of typical dispersions, which are stable over periods of 100 s of hours (Section S8, Fig. S13), are shown in Fig. 1D. Optical absorption spectra (Fig. 1E) show features expected for $\mathrm{MoS}_{2}$ and $\mathrm{WS}_{2}(20,21)$. In addition, a band edge at $\sim 5 \mathrm{eV}$ is clearly observed for dispersed BN. However, the spectra appear to be superimposed on a background, possibly due to scattering (Section S6, Fig. S4). A/l scaled linearly with concentration for all samples, allowing calculation of $\alpha$ values (Fig. 1F).

We performed TEM analysis on our dispersions, typically observing 2D flakes consisting of thin nanosheets. Examples of very thin sheets observed for all three materials are shown in Fig. 2A-C. The lateral size of these objects was typically $50-1000 \mathrm{~nm}$ for $\mathrm{MoS}_{2}$ and $\mathrm{WS}_{2}$ and 100-5000 $\mathrm{nm}$ for BN. (Section S7, Figs. S5-S12). We can examine these objects in more detail using aberration corrected TEM (Figs. 2D-F). These images and associated Fourier transforms illustrate the hexagonal symmetry of these materials. This is in contrast to reports on $\mathrm{MoS}_{2}$ and $\mathrm{WS}_{2}$ exfoliated by lithium intercalation which results in significant deviation from hexagonal structure (22-24). Fig. 2G-I show sections of the images in Fig. 2D-F after performing low-pass Butterworth filtering. These images reveal B-N bond lengths of $1.45 \AA$ and $\mathrm{MoS}_{2}$ and $\mathrm{WS}_{2}$ hexagon widths of $3.8 \AA$ and $4 \AA$, confirming that no distortions have been introduced by exfoliation. Analysis of TEM 
intensity profiles, coupled with flake edge analysis, electron diffraction and EELS data suggest the presence of mono-sheets in the sample(25).

Our dispersion/exfoliation method allowed us to prepare films of $\mathrm{BN}, \mathrm{MoS}_{2}$ and $\mathrm{WS}_{2}$ by vacuum filtration(17) or spraying, with thickness ranging from a few nm to hundreds of microns. Photographs of free-standing films are shown in Fig. 3A. Scanning electron and helium ion microscopy of the surface and edges of these films shows them to consist of partially aligned 2D platelets (Figs. 3B,C and S17). The versatility of the solventexfoliation method makes it simple to create hybrid dispersions and films simply by adding another material to the dispersion. We illustrated this by preparing hybrid films of $\mathrm{MoS}_{2}$ or $\mathrm{WS}_{2}$ mixed with graphene or single-walled nanotubes (SWNT) (Fig. 3D, E and Section S10). With the exception of pure BN, all films were mechanically robust (Section S10, Figs. S18, S20). Addition of graphene or SWNTs increased the DC conductivity, $\sigma_{\mathrm{DC}}$, from $\sim 10^{-6} \mathrm{~S} / \mathrm{m}$ for the TMD-only films to $\sim 2 \times 10^{4} \mathrm{~S} / \mathrm{m}$ for the SWNT based hybrids (Fig. 3F). We performed thermoelectric measurements on free-standing $\mathrm{WS}_{2} / \mathrm{SWNT}$ hybrid films, measuring $\sigma_{\mathrm{DC}}$ and the Seebeck coefficient, S. Obtaining significant increases in $\sigma_{D C}$ without degrading $S$ to give high power factor $\left(S^{2} \sigma_{D C}\right)$ is critically important in thermoelectric research(26). Here $S^{2} \sigma_{D C}$ increases from $0.2 \mu \mathrm{W} / \mathrm{K}^{2} \mathrm{~m}$ for disordered $\mathrm{WS}_{2}$ films (27) to $>100 \mu \mathrm{W} / \mathrm{K}^{2} \mathrm{~m}$ for $\mathrm{WS}_{2} / \mathrm{SWNT}$ films (Fig. 3G, Section S10).

Solvent processing greatly simplifies composite preparation (28-31), allowing us to prepare composites of polyurethane filled with $\mathrm{BN}, \mathrm{MoS}_{2}$ and $\mathrm{WS}_{2}$. We observed 
significant levels of reinforcement, comparable to the best results achieved using graphene(32) or nanoclays(33) as fillers (Figs. 3H, S19 and S20).

Exfoliated flakes can be deposited on substrates by spraying. Shown in Fig. 4A and B are an SEM and an AFM image of a silicon wafer spraycoated with $\mathrm{MoS}_{2}$. The objects observed are hundreds of nm wide in agreement with the TEM data (25). We can confirm the flakes consist of $\mathrm{MoS}_{2}$ by Raman mapping (Fig. 4C), based on the individual flake spectrum shown in Fig. 4D. We note that the peak positions $(25,34)$ in Fig. $4 \mathrm{D}$ are consistent with trigonal prismatic $(2 \mathrm{H})$ mono- or bi-layer $\mathrm{MoS}_{2}$ (Section S9). SEM analysis (Fig. 4 E-H) shows that while some deposited flakes are very thin, many are multilayers or clusters which have aggregated during deposition. AFM and STM imaging of individual flakes shows them to display typical thicknesses of $\sim 3-12 \mathrm{~nm}$ (Figs. $4 \mathrm{H}-\mathrm{J}$, S15, S16, Section S9). Some of these images, particularly the STM image in Fig. 4L, show steps. These are consistently $\sim 1 \mathrm{~nm}$ high and probably originate in layer edges. Electrical characterisation of individual flakes shows n-type field-effect behaviour characterised by mobilities of $\sim 0.01 \mathrm{~cm}^{2} / \mathrm{Vs}(9,10)$, rather lower than observed for mechanically exfoliated $\mathrm{MoS}_{2}$ flakes (Section S11).

This exfoliation process is not limited to $\mathrm{BN}, \mathrm{MoS}_{2}$ and $\mathrm{WS}_{2}$. We have exfoliated $\mathrm{MoSe}_{2}$, $\mathrm{MoTe}_{2}, \mathrm{TaSe}_{2}, \mathrm{NbSe}_{2}, \mathrm{NiTe}_{2}$ and $\mathrm{Bi}_{2} \mathrm{Te}_{3}$ in a number of the solvents in table S1 (Section S12, Figs. S23, S24), and believe similar solvents may exfoliate all $\mathrm{MX}_{2}$ compounds. We propose that this exfoliation technique is general as it can be applied to TMDs, graphene, $\mathrm{BN}$ and $\mathrm{Bi}_{2} \mathrm{Te}_{3}$. As such, we expect to extend it to $\mathrm{TMOs}(35)$ and other layered compounds. 
Figs
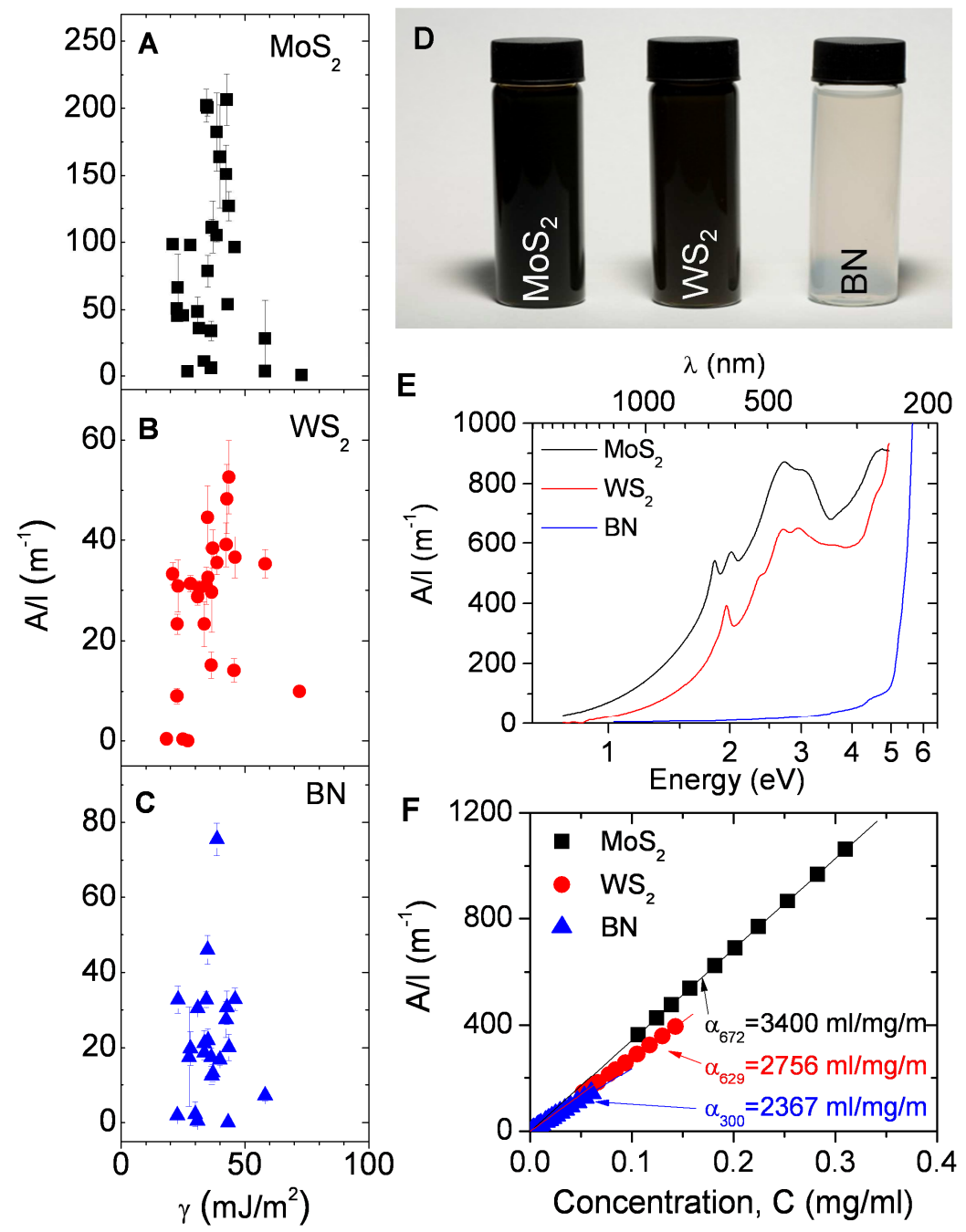

Fig. 1: Optical characterisation of nano-sheet dispersions. A), B) and C) Concentration remaining after centrifugation (plotted as $\mathrm{A} / \mathrm{l}$ ) for $\mathrm{MoS}_{2}, \mathrm{WS}_{2}$ and $\mathrm{BN}$ dispersed in a range of solvents, plotted versus solvent surface tension. D) Photographs of dispersions of $\mathrm{MoS}_{2}$ (in NMP), $\mathrm{WS}_{2}$ (in NMP) and $\mathrm{BN}$ (in IPA). E) Absorbance spectra of dispersions of $\mathrm{MoS}_{2}(\mathrm{NMP}, 0.16 \mathrm{mg} / \mathrm{ml}), \mathrm{WS}_{2}(\mathrm{NMP}, 0.15 \mathrm{mg} / \mathrm{ml})$ and $\mathrm{BN}(\mathrm{IPA}, 0.002$ 
$\mathrm{mg} / \mathrm{ml}$ ) F) Lambert-Beer plots for $\mathrm{MoS}_{2}$ (NMP), $\mathrm{WS}_{2}$ (NMP) and BN (IPA). The dispersions in D), E) and F) were prepared using optimised dispersion conditions (25).

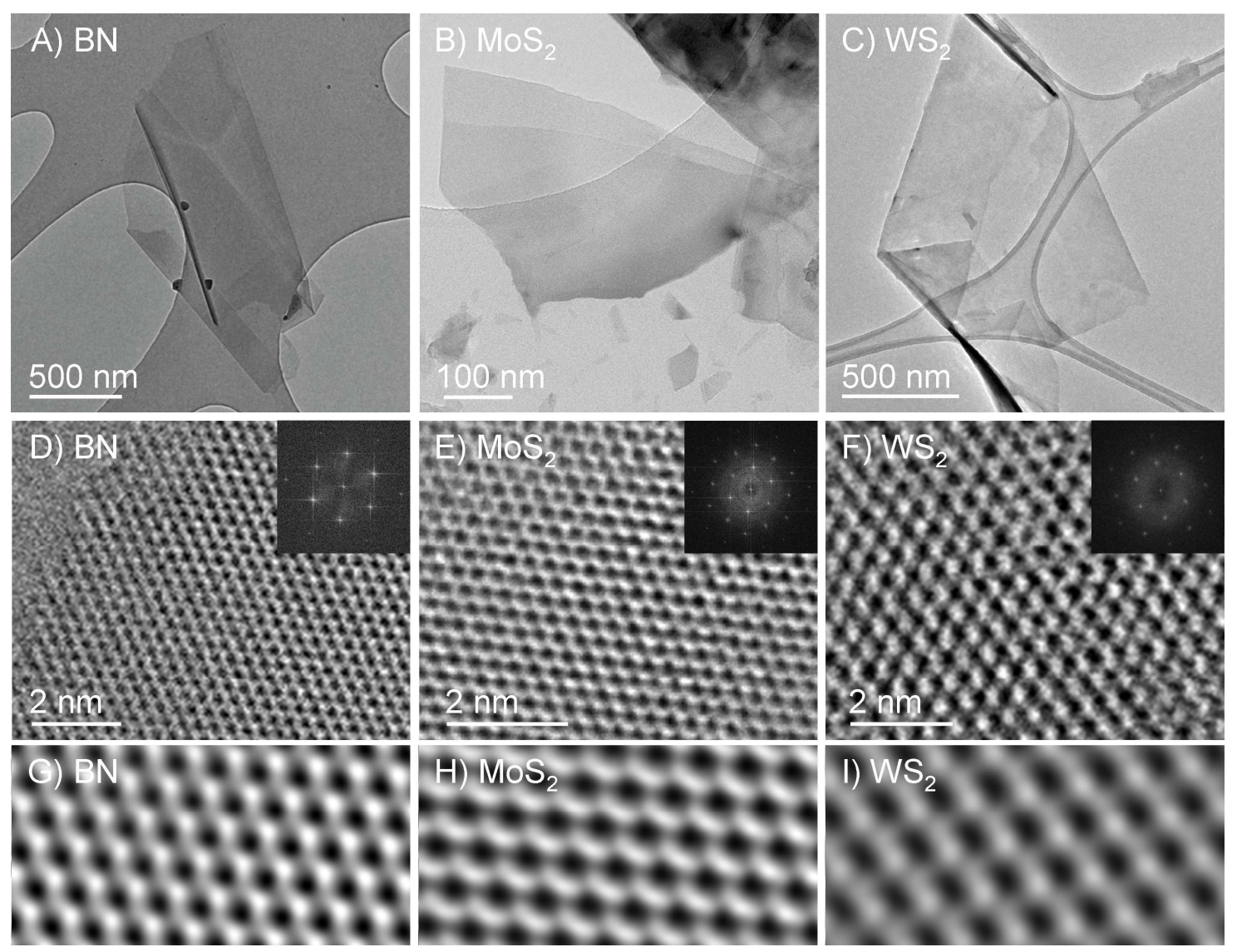

Fig. 2: TEM of nano-sheets. A), B) and C) Low resolution TEM images of flakes of BN, $\mathrm{MoS}_{2}$ and $\mathrm{WS}_{2}$ respectively. D), E) and F) High resolution TEM images of $\mathrm{BN}, \mathrm{MoS}_{2}$ and $\mathrm{WS}_{2}$ monolayers. Inset: Fast Fourier transforms of the images. G), H) and I) Butterworth filtered images of sections of the images in D), E) and F). 


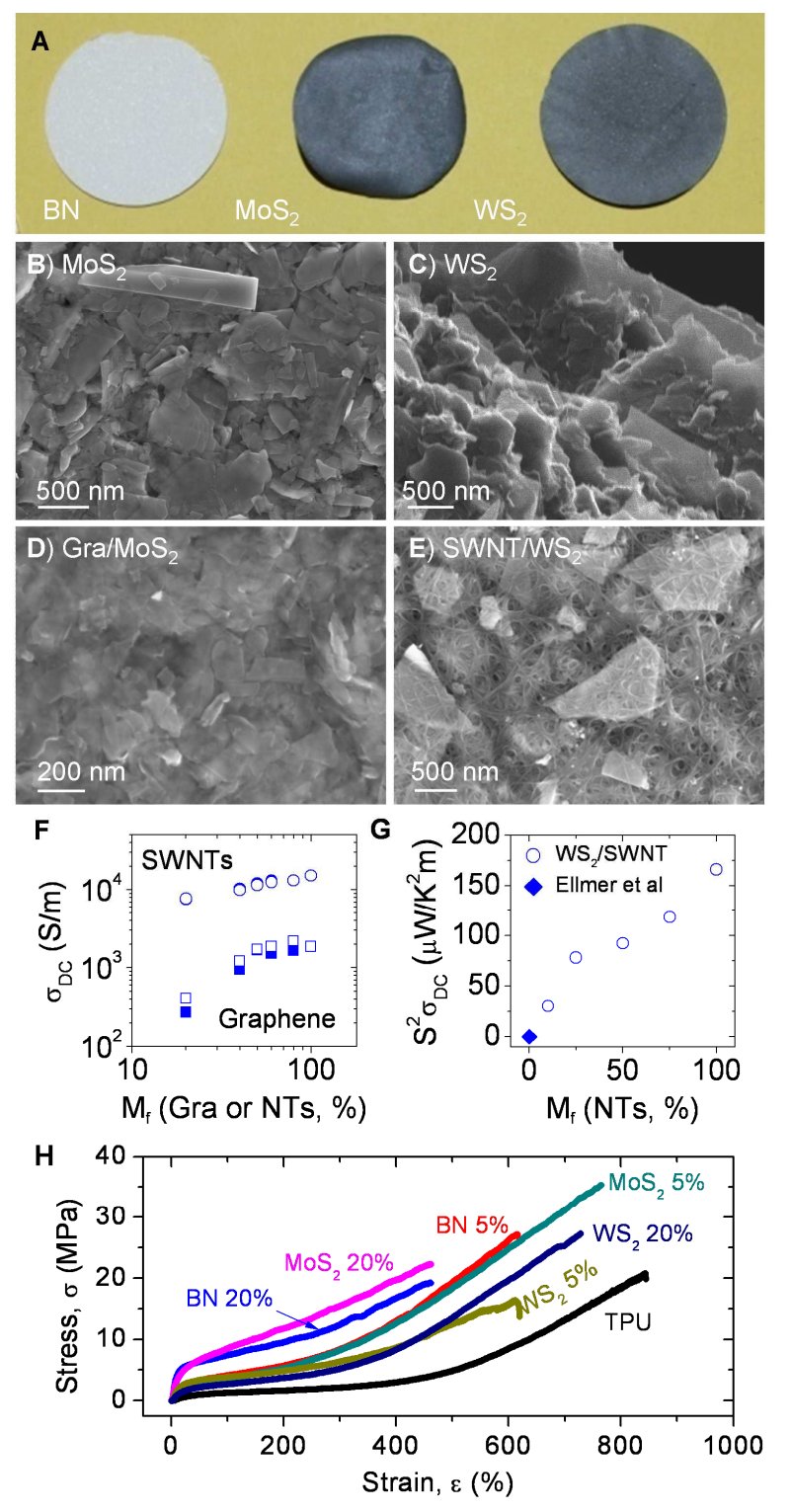

Fig. 3: Nano-sheet films, hybrids and composites. A) Photograph of free standing films of $\mathrm{BN}, \mathrm{MoS}_{2}$ and $\mathrm{WS}_{2}$ (thickness $\sim 50 \mu \mathrm{m}$ ). B) An SEM image of the surface of an $\mathrm{MoS}_{2}$ film. C) He ion microscope image of the edge of a $\mathrm{WS}_{2}$ film. D and E) SEM images of the surface of a graphene/MoS 2 hybrid film and a SWNT/ $\mathrm{WS}_{2}$ hybrid film respectively. F) DC conductivity of thin ( 200 nm) hybrid films prepared from mixtures of $\mathrm{WS}_{2}$ or $\mathrm{MoS}_{2}$ (open or closed symbols) and SWNTs or graphene (circles or squares). G) Product 
of Seebeck coefficient squared and DC conductivity (the power factor) for thick ( $\sim 50$ $\mu \mathrm{m}) \mathrm{WS}_{2} / \mathrm{SWNT}$ hybrid films. While the $\mathrm{WS}_{2}$ film proved too brittle to measure, Ellmer et al. measured $\mathrm{S}^{2} \sigma=0.22 \mu \mathrm{W} / \mathrm{K}^{2} \mathrm{~m}$ for a disordered $\mathrm{WS}_{2}$ film. In F) and $\left.\mathrm{G}\right)$, the $\mathrm{x}$-axis is the mass fraction of SWNTs or graphene. H) Representative stress-strain curves for composites of polyurethane filled with each layered compound at loading levels of $5 \mathrm{wt} \%$ and $20 \mathrm{wt} \%$. 

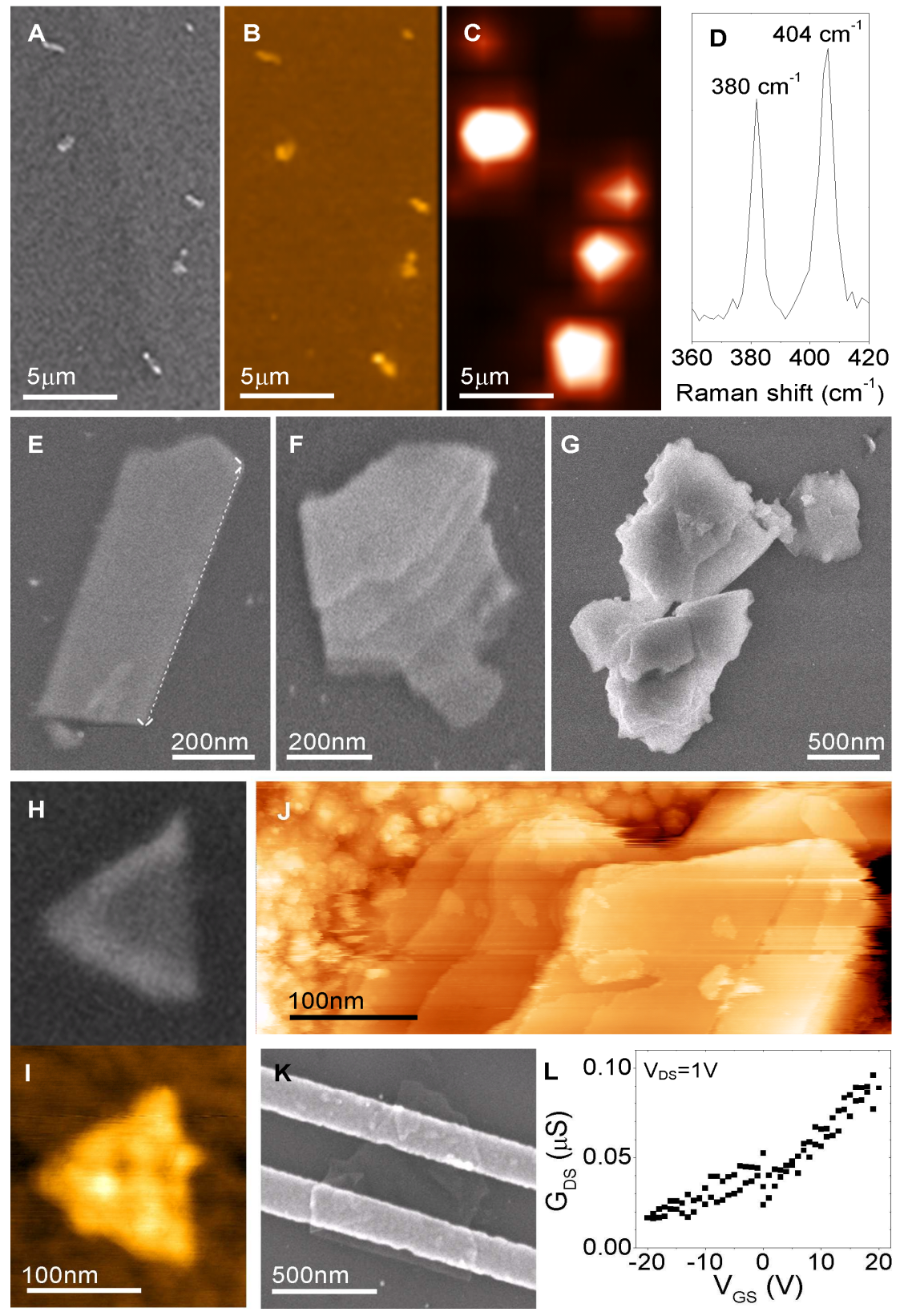

Fig. 4: Deposition of nano-sheets onto surfaces. A) and B) An SEM and an AFM image of $\mathrm{MoS}_{2}$ flakes deposited on $\mathrm{SiO}_{2}$ by spraying. C) A Raman map of the same region. D) Typical Raman spectrum of an individual flake. The Raman map plots the integral of the spectrum between 390 and $410 \mathrm{~cm}^{-1}$. E), F) and G) A very thin flake, a multilayer and a 
cluster of aggregated multilayers. The dashed line in $\mathrm{E}$ has been inserted to illustrate the straightness of the flake edge. H) and I) SEM and AFM images of an individual flake. J) An STM image of an individual flake. The flakes in I) and J) have heights of 5 and $10 \mathrm{~nm}$ respectively.(25) K) An SEM image of an $\mathrm{MoS}_{2}$ flake on $\mathrm{Si} / \mathrm{SiO}_{2}$ with electrodes deposited on top. L) Source-drain conductance as a function of gate voltage for the flake shown 
References

1. S. K. Mishra, S. Satpathy, O. Jepsen, Journal of Physics-Condensed Matter 9, 461 (1997).

2. $\quad$ E. A. Marseglia, International Reviews in Physical Chemistry 3, 177 (1983).

3. J. A. Wilson, A. D. Yoffe, Advances in Physics 18, 193 (1969).

4. $\quad$ F. R. Gamble, B. G. Silbernagel, Journal of Chemical Physics 63, 2544 (1975).

5. $\quad$ F. Clerc et al., Journal of Physics-Condensed Matter 19, 17 (2007).

6. A. K. Geim, Science 324, 1530 (2009).

7. B. Poudel et al., Science 320, 634 (2008).

8. $\quad$ A. Splendiani et al., Nano Letters 10, 1271 (2010).

9. K. S. Novoselov et al., Proceedings of the National Academy of Sciences of the United States of America 102, 10451 (2005).

10. A. Ayari, E. Cobas, O. Ogundadegbe, M. S. Fuhrer, Journal of Applied Physics 101, (2007).

11. R. Ruoff, Nature Nanotechnology 3, 10 (2008).

12. P. Joensen, R. F. Frindt, S. R. Morrison, Materials Research Bulletin 21, 457 (1986).

13. C. Liu, O. Singh, P. Joensen, A. E. Curzon, R. F. Frindt, Thin Solid Films 113, 165 (1984).

14. H. Matte et al., Angewandte Chemie-International Edition 49, 4059 (2010).

15. A. Nag et al., ACS Nano 4, 1539 (2010).

16. C. Y. Zhi, Y. Bando, C. C. Tang, H. Kuwahara, D. Golberg, Advanced Materials 21, 2889 (2009).

17. Y. Hernandez et al., Nature Nanotechnology 3, 563 (2008).

18. S. D. Bergin et al., Advanced Materials 20, 1876 (2008).

19. S. D. Bergin et al., ACS Nano 3, 2340 (2009).

20. E. A. Ponomarev, M. NeumannSpallart, G. Hodes, C. LevyClement, Thin Solid Films 280, 86 (1996).

21. J. P. Wilcoxon, P. P. Newcomer, G. A. Samara, Journal of Applied Physics 81, 7934 (1997).

22. R. Bissessur, M. G. Kanatzidis, J. L. Schindler, C. R. Kannewurf, Journal of the Chemical Society-Chemical Communications, 1582 (1993).

23. G. L. Frey, K. J. Reynolds, R. H. Friend, H. Cohen, Y. Feldman, Journal of the American Chemical Society 125, 5998 (2003).

24. R. A. Gordon, D. Yang, E. D. Crozier, D. T. Jiang, R. F. Frindt, Physical Review $B$ 65, (2002).

25. See supporting material on Science Online.

26. M. S. Dresselhaus et al., Advanced Materials 19, 1043 (2007).

27. K. Ellmer, C. Stock, K. Diesner, I. Sieber, Journal of Crystal Growth 182, 389 (1997).

28. E. Benavente, M. A. S. Ana, G. González, physica status solidi (b) 241, 2444 (2004). 
29. R. F. Frindt, D. Yang, Molecular Crystals and Liquid Crystals Science and Technology Section a-Molecular Crystals and Liquid Crystals 311, 367 (1998).

30. J. P. Lemmon, J. H. Wu, C. Oriakhi, M. M. Lerner, Electrochimica Acta 40, 2245 (1995).

31. B. H. Xu, B. Z. Lin, D. Y. Sun, C. Ding, Electrochimica Acta 52, 3028 (2007).

32. U. Khan, P. May, A. O'Neill, J. N. Coleman, Carbon 48, 4035 (2010).

33. S. M. Liff, N. Kumar, G. H. McKinley, Nature Materials 6, 76 (2007).

34. C. Lee et al., ACS Nano 4, 2695 (2010).

35. M. Osada, T. Sasaki, Journal of Materials Chemistry 19, 2503 (2009).

36 The authors would like to acknowledge IRCSET (Embark Initiative), the European Research Council and Science Foundation Ireland (grant number 07/IN.7/I1772), for financial support. VN would like to acknowledge funding from the Marie Curie grant PIEF-GA-2008-220150 and the Royal Academy of Engineering/EPSRC and thank BegbrokeNano for their provision of research facilities. 